\title{
Reflexiones sobre la Cultura Científica y Tecnológica desde el enfoque CTS.
}

Rreflections on Culture Science and

Technology from the STS.

Fecha de Recepción

8 de febrero de 2011
Reflexões sobre a Cultura, Ciência e Tecnologia do CTS.

Aceptado para su publicación

II de mayo de 20 I I

\section{Rolando Pablo Alejandro Júarez}

Profesor Titular. Cátedra Fisiología Humana. FOUNNE.

Sandra Elena Martínez Profesora Adjunta. Cátedra Práctica

Clínica Preventiva I. FOUNNE.

\section{Jorge Orlando Ponce}

Auxiliar Docente $I^{\circ}$. Cátedra Fisiología Humana. FOUNNE.

Alejandro Joaquín Encina Tutuy Adscripto. Cátedra Práctica Clínica Preventiva I.

FOUNNE.

\section{Resumen}

CTS incorpora la perspectiva de las disciplinas sociales, en los ámbitos de la investigación académica, la educación y la política pública, para entender el proceso de la actividad científica y tecnológica. Promueve la alfabetización científica y forma parte de la cultura general en las sociedades democráticas modernas.

Esta publicación desarrolla una revisión en la temática, centrándose en la cultura científica, analizando su interacción con la percepción social de la ciencia y la participación ciudadana.

\section{Palabras Claves}

Cultura científica, percepción social de la ciencia, participación ciudadana.

\section{Summary}

STS incorporates the perspective of social science, in the areas of academic research, education and public policy, to understand the process of scientific and technological activity. Promoting scientific literacy is part of the general culture in modern democratic societies.

This publication develops a review on the subject, focusing on scientific literacy, analyzing its interaction with the social perception of science and public participation.

\section{Keywords}

Scientific culture, social perception of science, public participation. 


\section{Resumo}

CTS incorpora a perspectiva das ciências sociais nas áreas da pesquisa acadêmica, educação e políticas públicas, para entender o processo da atividade científica e tecnológica. Promover a literacia científica é parte da cultura geral nas sociedades democráticas modernas.

Esta publicação desenvolve uma análise sobre o assunto, com foco na alfabetização científica, analisando sua interação com a percepção social da ciência e da participação cidadã.

\section{Palavras chave \\ Cultura científica, a percepção social da ciência, participação cidadã.}

\section{Introducción}

La expresión "Ciencia, Tecnología y Sociedad" (CTS) suele definir un ámbito de trabajo académico, cuyo objeto de estudio está constituido por los aspectos sociales de la ciencia y la tecnología (CyT), tanto en lo que concierne a los factores sociales que influyen sobre el cambio científico-tecnológico, como en lo que ateñe a las consecuencias sociales y ambientales, (García Palacios y col., 200I).

Se origina en Europa a partir de la confluencia de la sociología de la ciencia, que con un enfoque institucional desarrolló Merton a partir de los años 30, por un lado, y por el otro, de la relación entre ciencia y poder puesta de relieve por Bernal en los mismos años, como así también los desarrollos de Solla Price reclamando un enfoque interdisciplinario que postulaba una "ciencia de la ciencia", (Vaccarezza, 1998).

Desde el punto de vista del enfoque CTS, la conceptualización de la ciencia no debe reducirse exclusivamente a lo que las comunidades de científicos pueden decir de ella (Varela, 1998); donde CyT son presentadas como formas autónomas de la cultura, como actividades valorativamente neutrales, como una alianza heroica de conquista de la naturaleza, (Echeverría, 1995).

Deberían incorporarse, además, las visiones que "desde fuera" se construyen sobre la ciencia (sus prácticas y sus agentes). La imagen de la ciencia que produce la sociedad se concebiría como un espectro culturalmente situado de representaciones varias, aunque heurísticamente se pudiera realizar un ejercicio de jerarquía conceptual, el cual sería, asimismo, una construcción localizada culturalmente, (Ayús Reyes, 2002).

Esta noción se comprende ubicándola sobre el fondo de las tendencias construccionistas, contextualizadoras y relativistas que pugnan por dialogar y posicionarse en el debate epistemológico y político, al menos desde la década de los ' 60 hasta nuestros días, (Quiroz Pérez, 2010). La clave se encuentra en presentar la ciencia-tecnología no como un proceso o actividad autónoma que sigue una lógica interna de desarrollo en su funcionamiento óptimo, sino como un proceso o producto inherentemente social donde los elementos no técnicos (por ejemplo, valores morales, convicciones religiosas, intereses profesionales, presiones económicas, etc.) desempeñan un papel decisivo en su génesis y consolidación, (López Cerezo, 1998).

En América Latina, la CyT, se caracterizan por: a) escasa magnitud; b) desvinculación con la sociedad; c) dependencia de la actividad científica realizada en el mundo desarrollado. El conocimiento científico que se produce tiene poca o ninguna relación con la realidad social, económica y cultural de nuestros países, razón por la cual no sólo está desvinculada, sino que también pierde legitimidad, (Salas Madriz, 2007).

El objetivo de esta publicación es mostrar el carácter de objetivo social prioritario de la cultura científica, discutiendo los mecanismos de relación entre ciencia y sociedad.

\section{Dimensión CTS del Currículo de Ciencias}

El enfoque CTS ha potenciado para la mejora de la enseñanza de las ciencias y su innovación, aportaciones a las que algunos autores prefieren denominar la dimensión CTS del currículo de ciencias, (Solbes, Vilches y Gil, 2002).

Las contribuciones del enfoque CTS, frente a los contenidos sustantivos tradicionales de los currículos de ciencias y tecnologías, son la enseñanza de: a) los escenarios histórico y social en el que se han gestado los conocimientos científicos; b) la naturaleza de la ciencia (qué es la ciencia, su funcionamiento interno y externo, cómo se construye y desarrolla el conocimiento que produce, los métodos que usa para validar este conocimiento, los valores implicados en las 
actividades científicas, la naturaleza de la comunidad científica, los vínculos con la tecnología, las relaciones de la sociedad con el sistema tecnocientífico $y$, viceversa, las aportaciones de éste a la cultura y al progreso de la sociedad); c) la relación entre los conocimientos científicos con el contexto tecnológico y sus correspondientes desarrollos e innovaciones; d) la conexión entre los conocimientos científicos universitarios con las controversias sociales y medioambientales del presente; e) los principales problemas que tiene la humanidad para un futuro más sostenible, (Acevedo y col., 2005).

La esencia del papel de CTS en la educación científica está sobre todo en educar para la participación cívica en las decisiones tecnocientíficas, una finalidad educativa clave de la enseñanza de las ciencias que da sentido pleno al lema de alfabetización científica y tecnológica para todas las personas $y$, a la vez, potencia las actitudes democráticas, (Acevedo, 2004; Martín, 2004). No es extraño, por ello, que se haya llegado a establecer una analogía entre la alfabetización básica iniciada el siglo pasado y el actual movimiento de alfabetización científica y tecnológica, (Fourez, 1997).

La ciencia y la tecnología han dejado de ser parte del discurso de unos pocos académicos para formar parte de los conocimientos básicos del ciudadano común. De hecho, para interpretar las noticias diarias se requiere de un conocimiento mínimo en CyT, (Cajas, 200I).

Nos hallamos, por tanto, frente a un amplio reconocimiento de la necesidad de una alfabetización científica, expresión, ampliamente utilizada en la actualidad y en cuyo significado conviene detenerse.

\section{Alfabetización Científica y Tecnológica}

Para identificar y medir la comprensión de una población en relación con la CyT, se utiliza un concepto, la "alfabetización científica", término que no tiene una definición precisa. El concepto de alfabetización surgió durante el siglo $\mathrm{XIX}$, junto con la conciencia sobre la necesidad de que las personas supieran leer y escribir, para desenvolverse en el mundo laboral. En la década del setenta del siglo $X X$, los pedagogos comenzaron a hablar de "analfabetos funcionales", categoría que continua vigente en nuestros días. Son aquellas personas sin competencias mínimas para desenvolverse en las sociedades actuales, como utilizar un cajero automático o una computadora, (Miller, Pardo y Niwa, 1999).

Bybee (1997) sugiere rechazar la simplificación inapropiada del concepto a su significado literal: una alfabetización científica; aunque ha de incluir el manejo del vocabulario científico, no debe limitarse a esa definición funcional. Propone la expresion "alfabetización científico-tecnológica multidimensional”, señalando que se extiende más allá del vocabulario, de los esquemas conceptuales y de los métodos procedimentales, para incluir otras dimensiones de la ciencia. Propone ayudar a los estudiantes a desarrollar perspectivas de la CyT que incluyan la historia de las ideas científicas, la naturaleza de la CyT y el papel de ambas en la vida personal y social.

En opinión de Gil Pérez y col. (2005), hablar de alfabetización científica, de ciencia para todos, supone pensar en un mismo currículo básico para todos los estudiantes, que convierten a la educación científica en parte de una educación general y requiere estrategias que eviten las repercusiones de las desigualdades sociales en el ámbito educativo.

Fensham (2002a, 2002b), fustiga la posibilidad de lograr una sociedad científicamente alfabetizada a través de la educación científica escolar, pues constituye una ilusión que ignora la complejidad de los conceptos científicos implicados. Es absolutamente irrealista, añade, creer que este nivel de conocimientos pueda ser adquirido, ni siquiera en las mejores escuelas.

Estas críticas nos obligan, a reflexionar que la alfabetización científica es parte de una cultura científica para toda la ciudadanía, donde debemos poner énfasis en las relaciones CTS, con vistas a favorecer la participación ciudadana en la toma fundamentada de decisiones, (Aikenhead, 1985). Es deseable que la "alfabetización científica" de una persona crezca a lo largo de toda su vida, entonces, la vía educativa formal propia de una enseñanza reglada no puede ser el único responsable, debiendo existir otras instancias que pueden contribuir a completarla e incrementarla: los medios de comunicación de masa, (Sjøberg, 2003).

\section{Cultura Científica y Tecnológica}

La definición de cultura científica ha sido una 
preocupación constante en muchos países iberoamericanos, y particularmente en aquellos que adquieren conciencia de sus deficiencias científico-técnicas respecto a los países más desarrollados. La acepción general de cultura científica está vinculada con el tipo de relación que se establece entre la ciencia y la sociedad y la metodología asociada a su valoración, (Márquez Nerey y Tirado Segura, 2009).

La cultura científica entendida de forma activa supone un tipo de conciencia adquirida por los ciudadanos cuando se ven involucrados en decisiones personales o problemas sociales relacionados con la aplicación de la ciencia y el desarrollo tecnológico. Por ejemplo, cuando una asociación de vecinos busca, obtiene y utiliza información sobre efectos biológicos de la radiación electromagnética a la hora de considerar críticamente la posible instalación de una antena de telefonía celular sobre el tejado de su edificio o en el terreno baldío de al lado, (MCYT, 2004).

Para Zamarrón (2006), una persona con cultura científica requiere contar con información pero también con una preparación y habilidades que le permitan situar el conocimiento en su esencia y su sentido. Entendemos que esto significa una capacidad de análisis y contextualización de lo que sucede en el mundo de la ciencia.

La cultura científica no es un atributo de individuos sino de sociedades, $y$ aunque las sociedades están formadas por individuos, no se podría afirmar que cada individuo "representa" a la sociedad $y$, por lo tanto, al conjunto de su cultura, sino que cada uno de ellos mantiene una relación con la sociedad que es irreductible tanto a la sociedad como al individuo. En este sentido, la cultura de la ciencia y la tecnología no consiste solamente en un stock de conocimientos codificados que incorpora el individuo sino que también implica otras dimensiones no menos relevantes, (OEI/ RICYT, 2003).

Así, la definición de Leonardo Vaccarezza (2008) nos parece apropiada, pues involucra una amplitud de elementos en juego: "Se entiende la cultura científica como comprensión de la dinámica social de la ciencia, de manera que se tejen, en una interrelación entre productores de conocimientos científicos y otros grupos sociales, todos ellos como partícipes del devenir de la cultura, produciendo significados cuyos orígenes y justificaciones provienen desde distintas prácticas, inte- reses, códigos normativos y relaciones de poder, entendiéndose como un devenir continuo."

Es decir, una sociedad estará más o menos cientizada en la medida en que la cuestión de "hacia dónde se dirige la CyT" se constituye en un tópico de la cultura general y el debate social.

Es necesario distinguir el concepto de cultura cientifica de las significaciones de "percepción pública de la CyT" y "participación ciudadana". Aunque, parten de esquemas interpretativos $y$ tradiciones cognitivas diversas, se encuentran estrechamente asociados, por lo que su tratamiento conjunto conduce al análisis y evaluación de la cultura científica de una sociedad en particular.

El concepto de percepción pública remite al proceso y mecanismos de comunicación social y al impacto de éstos sobre la formación de contenidos, actitudes y expectativas de los miembros de la sociedad sobre la CyT. Mientras que la participación pública ayuda a encaminar el desarrollo tecnocientífico en direcciones consideradas relevantes por la sociedad.

La percepción pública de la CyT está ocupando un sitio preponderante de la agenda políticosocial de numerosos países, incorporarando estas cuestiones como un elemento central en la elaboración de políticas públicas, (Velazco y col., 2006).

La encuesta, dada su capacidad para explorar las opiniones distribuidas en la población, se transformó en un instrumento importante en los estudios sobre la conciencia pública de la ciencia.

La tendencia internacional señala que la aplicación periódica de las encuestas permite: a) registrar cambios y evoluciones en la cultura científica de la sociedad y del impacto de las políticas públicas específicas; b) la periodicidad crea cultura ciudadana, pues la sociedad se familiariza paulatinamente con el ámbito científico y tecnológico, hecho importante para un país como la Argentina que sufrió décadas de desmantelamiento de las estructuras científico-tecnológicas, (SeCyT/ ONCTIP, 2007).

El análisis de contenidos de los medios masivos de comunicación es otra metodología empleada en los países desarrollados para el estudio de la "cultura científica", como complemento de las encuestas, (Polino, Fazio, Vaccarezza, 2003). 


\section{Reflexiones Finales}

La CyT son instituciones sociales que han configurado nuestra civilización. La civilización occidental globalizada es una civilización científica y tecnológica. Por ende, la sociedad debe participar en su diseño y realización.

La CyT no están desconectadas de las determinaciones culturales de la sociedad global donde actúan. Pero a la sociedad le cuesta por sí misma comprender que CyT deben concebirse y practicarse como parte de la cultura cotidiana.

La cultura científica y tecnológica es un reto social que interpela diversos actores sociales: científicos y tecnólogos, políticos y educadores, y profesionales de la comunicación, convocados para formar ciudadanos capaces de intervenir más y mejor en las decisiones concernientes a la CyT contemporáneas.

Por tanto, más allá de la incorporación de una dimensión CTS añadida a la enseñanza de las ciencias, son necesarios otros procesos de construcción de la cultura científica de la sociedad: comunicación de los resultados y valores científicos, conocimiento popular, percepción social de las controversias entre expertos y la construcción social de interpretaciones sobre distintos aspectos relativos al conocimiento mismo.

Es decir, la cultura científica va más allá que la "alfabetización" en ciencia, trasciende el acopio de información que podamos adquirir. Más que el conocimiento, la comprensión de la ciencia como producción intelectual y social, son fundamentales para pensar en la cultura científica del ciudadano del siglo XXI.

El denominador común de estas propuestas indica que, por un lado, toda la sociedad debe ser partícipe de los logros científicos y, por otro lado, que toda la sociedad debe estar en condiciones de discutir los dilemas que la investigación científica plantea. Es justamente la participación de la ciudadanía en las decisiones sociales tecnocientíficas lo que el enfoque propio del movimiento CTS reivindica como principal finalidad para la educación científica.

La percepción que la sociedad tenga de los científicos, las instituciones científicas y los resultados del conocimiento, estará determinada por las formas en que las políticas de ciencia y tecnología integren a la sociedad en el sistema científico- tecnológico, mediante la solución de problemas sociales concretos.

\section{Bibliografía Consultada}

I. Acevedo J. A. (2004). Reflexiones sobre las finalidades de la enseñanza de las ciencias: Educación científica para la ciudadanía. Revista Eureka sobre Enseñanza y Divulgación de las Ciencias, I (I): $3-16$.

2. Acevedo J. A., Vázquez A., Martín M., Oliva J. M., Acevedo P., Paixão M. F. y Manassero M. A. (2005). Naturaleza de la ciencia y educación científica para la participación ciudadana. Una revisión crítica. Revista Eureka sobre Enseñanza y Divulgación de las Ciencias, 2 (2): 121 - 140.

3. Aikenhead G. S. (1985). Collective decision making in the social context of science. Science Education, 69 (4): 453 - 475.

4. Ayús Reyes R (2002). Estudios sociales de ciencia y tecnología: merodeando en el campo. CTS+I. Sala de lectura. Organización de Estados Iberoamericanos para la Educación, la Ciencia y la Cultura. Disponible en: http://www.campus-oei. org/salactsi/ramfis.htm [15 pp.; acceso: 10 enero de 20II].

5. Bybee R (1997). Towards an Understanding of Scientific Literacy. En Graeber, W. y Bolte, C. (Eds.), Scientific Literacy. Kiel: IPN.

6. Cajas F (200I). Alfabetización científica y tecnológica: la transposición didáctica del conocimiento tecnológico. Enseñanza de las ciencias, 19 (2): 243 $-254$.

7. Echeverría J. (1995). Filosofía de la ciencia. Madrid: Akal.

8. Fensham P. J. (2002a). Time to change Drivers for Scientific Literacy. Canadian Journal of Science, Mathematics and Technology Education, 2 (I): 9 - 24.

9. Fensham P. J. (2002b). De nouveaux guides pour l'alphabétisation scientifique. Canadian Journal of Science, Mathematics and Technology Education, 2 (2): 133 - 149.

10. Fourez G. (1997). Alfabetización científica y tecnológica. Acerca de las finalidades de la enseñanza de las ciencias. Buenos Aires: Colihue.

II. García Palacios E. M., González Galbarte J. C., López Cerezo A., Luján J. L., Martín Gordillo M., Osorio C., Valdés C. (200I). Ciencia, Tecnología y Sociedad: una aproximación conceptual. Cuadernos de Iberoamérica. Madrid: Organización de Estados Iberoamericanos para la Educación, la Ciencia y la Cultura (OEI).

12. Gil Pérez D., Sifredo C., Valdés P., Vilches A. (2005). ¿Cuál es la importancia de la educación 
científica en la sociedad actual? En: Gil- Pérez, D., Macedo, B., Martínez Torregrosa, J., Sifredo, C., Valdés, P. y Vilches, A. (Eds.). ¿Cómo promover el interés por la cultura científica? Una propuesta didáctica fundamentada para la educación científica de jóvenes de 15 a 18 años. Santiago: OREALC/UNESCO.

13. López Cerezo J. A. (1998). Ciencia, Tecnología y Sociedad: el estado de la cuestión en Europa y Estados Unidos. Revista Iberoamericana de Educación; |8: 41 - 68.

14. Márquez Nerey E., Tirado Segura F. (2009). Percepción social de la ciencia y la tecnología de adolescentes mexicanos. Revista lberoamericana de Ciencia, Tecnología y Sociedad, 2: 17 - 34.

15. Martín M. (2004). Educar per participar en ciència $i$ tecnologia. Revista del Col.legi Oficial de Doctors i Llicenciats en Filosofia i Lletres i en Ciències de Catalunya, 122: 57-70.

16. Miller J., Pardo R. y Niwa F. (1999). Percepciones del Público ante la Ciencia y la Tecnología. Estudio comparativo de la Unión Europea, Estados Unidos, Japón y Canadá. Fundación BBV: Bilbao, España.

17. MCyT (2004). Primera Encuesta Nacional de Percepción Pública de la Ciencia, Cultura Científica y Participación Ciudadana. Venezuela: Ministerio de Ciencia y Tecnologia.

18. OEI/RICYT (2003). Proyecto Iberoamericano de Indicadores de percepción pública, cultura científica y participación ciudadana (200I-2003). Organización de Estados Iberoamericanos para la Educación, la Ciencia y la Cultura (OEI) y Red Iberoamericana de Indicadores de Ciencia y Tecnología (RICYT) del Programa CYTED. Revista Iberoamericana de Ciencia, Tecnología, Sociedad e Innovación. Disponible en: http:// www.observatorio.mincyt.gov.ar/docs/ percep/ encuestapercepcion.pdf, [acceso 20 de enero de 20II].

19. Polino C., Fazio M. E., Vaccarezza L. (2003). Medir la percepción pública de la ciencia en los países iberoamericanos. Aproximación a problemas conceptuales. Revista Iberoamericana de Ciencia, Tecnología, Sociedad e Innovación [online]. Número 5 / Enero - Abril. Disponible en: http:// www.oei.es/revistactsi/ numero5/articulol.htm, [acceso 4 de julio de 20I0].

20. Quiroz Pérez A. M., Aristizábal Berrío W. J., L. H. Lopera Lopera (2010). Apuntes para una crítica de la racionalidad tecnocientífica desde el enfoque Phronético. Revista Ciencia Tecnología Sociedad, 2: I|3-12I.

2I. Salas Madriz F. E. (2007). Educación e investigación y desarrollo en América Latina. Los últimos treinta años. Educación (Universidad de Costa
Rica), 3I (2): 29-43.

22. SeCyT/ONCTIP (2007). La Percepción de los Argentinos sobre la Investigación Científica en el País. Segunda Encuesta Nacional de Percepción Pública de la Ciencia. Secretaría de Ciencia, Tecnología e Innovación Productiva (SeCyT). Observatorio Nacional de Ciencia, Tecnología e Innovación Productiva (ONCTIP). Ministerio de Educación, Ciencia y Tecnología. Disponible en: http://www.observatorio.mincyt.gov.ar/docs/ percep/Informe_Final_2007. pdf, [acceso 22 de junio de 20I0].

23. Sjøberg S (2003). Science and Technology Education: Current Challenges and Possible Solutions. En E.W. Jenkins (Ed.): Innovations in science and technology education, Vol. VIII. París: UNESCO.

24. Solbes J, Vilches A y Gil D (2002). Papel de las interacciones $C T S$ en el futuro de la enseñanza de las ciencias. En P. Membiela (Ed.): Enseñanza de las ciencias desde la perspectiva ciencia-tecnologíasociedad. Formación científica para la ciudadanía. Madrid: Narcea.

25. Vaccarezza LS (1998). Ciencia, Tecnología y Sociedad: el estado de la cuestión en América Latina. Revista Iberoamericana de Educación (Madrid, OEI), 18:13-40.

26. Vaccarezza LS (2008). Exploraciones en torno al concepto de cultura científica. En FECYT, Resúmenes del Congreso Iberoamericano de Ciudadanía y Políticas Públicas de Ciencia y Tecnología. Madrid, p. II0.

27. Varela FJ (1998). Conocer. Las ciencias cognitivas: tendencias y perspectivas. Cartografía de las ideas actuales. Gedisa: Barcelona, España.

28. Velazco E, Callieri C, Iragui J, Palmeyro E, Venturuzzi G, Mazzei H, et al. (2006). Hacia una Agenda Nacional en Materia de Competitividad. Observatorio de Políticas Públicas - Jefatura de Gabinete de Ministros, República Argentina. Disponible en: http://www.sgp.gov.ar/contenidos/ag/ paginas/opp/docs/2006/03_OPP_2006_ COMPETITIVIDAD.pdf, [acceso 2 de julio de 2010].

29. Zamarron $G$ (2006). De cultura científica y anexas. En: Universidad de Baja California y Mario Porrúa Edits. Universidad, comunicación y ciencia: contrastes. México. 